\title{
Fluorination: An Effective Molecular Design Strategy for Efficient Photovoltaic Materials
}

\author{
DENG Dan, ZHOU Erjun *, WEI Zhixiang * \\ National Center for Nanoscience and Technology, Beijing 100190, P. R. China.
}

\begin{abstract}
Organic solar cells (OSCs) have received widespread attention for their advantages of cheap, light, flexible characteristics and roll-to-roll printing technology. However, the efficiencies of OSCs are still lower than $50 \%$ of the theoretical Shockley-Queisser detailed-balance efficiency limit. Consequently, to further improve device performance, it is significant to develop molecular design strategies to lower the energy loss and enhance the utilization of absorbed photons. From the molecular design aspects, down-shifting energy levels is an effective way to lowering the energy loss in order to obtain a high open circuit voltage, and optimizing

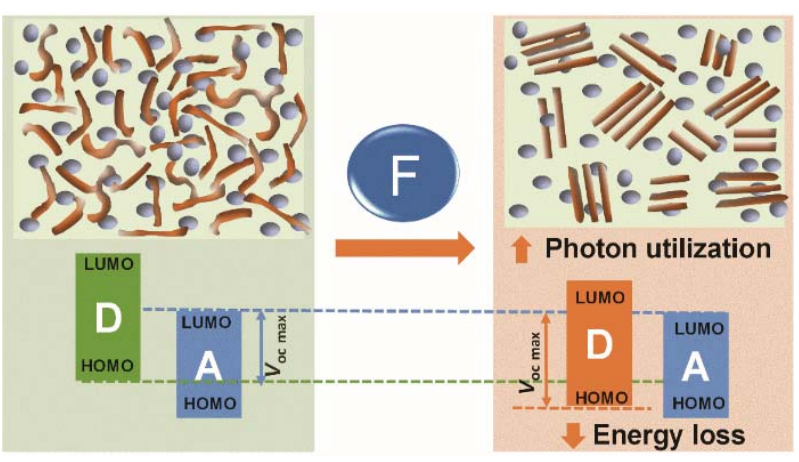
the morphology is an efficient approach to lowering the fill factor and current density loss. Introduction of fluorine atom in molecules is an effective molecular design strategy to realize both above-mentioned requirements. In this review, starting from the characteristics of fluorine atoms, we summarized the fluorination effects on adjusting molecular levels. Whether the fluorine attached to the donor units, acceptor units or $\pi$-bridge units, it could efficiently downshift the energy levels. However, fluorinating the molecular backbone affects the energy levels more significantly than fluorinating the side chains of the two-dimensional structures. The introduction of fluorine is also an effective approach to optimize molecular packing and morphology. Generally, whether the fluorine attached to the donor units, acceptor units or $\pi$-bridge units, it can effectively increase molecular coherence length, decrease $\pi-\pi$ stacking distance, and enhance domain purity. However, there is a saturation of the fluorine on the backbone, further introduction of the fluorine can accelerate molecular aggregation and induce disorder. In addition, the position of fluorination is important. In this review, we also briefly discuss the fluorination strategy for representative and high-efficiency photovoltaic material designs, including small molecule, polymer, and non-fullerene OSCs, mainly focusing on improving efficiency by reducing the efficiency losses. Fluorination is advantageous only for OSCs with high HOMO energy levels or poor molecular packing; otherwise, it can compromise device performance. OSCs based on narrow band-gap non-fullerene acceptors with low energy loss show promise for highly efficient device performance. Fluorination provides an effective means to fine-tune energy levels and form ideal microstructures to further reduce the efficiency loss and achieve a breakthrough in device performance.
\end{abstract}

Key Words: Fluorination; Energy level; Morphology; Energy loss; Power conversion efficiency

Received: February 23, 2018; Revised: March 22, 2018; Accepted: March 23, 2018; Published online: March 27, 2018.

*Corresponding authors. Email: weizx@nanoctr.cn (W.Z.); zhouej@nanoctr.cn (Z.E.). Tel.: +86-010-82545565.

The project was supported by the National Natural Science Foundation of China (51603051, 21125420) and the Youth Innovation Promotion Association CAS. 国家自然科学基金 $(51603051 ， 21125420)$ 和中国科学院青年创新促进会资助项目

(C) Editorial office of Acta Physico-Chimica Sinica 


\title{
氟化策略：高效有机光伏材料的设计与应用
}

\author{
邓丹, 周二军 ${ }^{*}$, 魏志祥* \\ 国家纳米科学中心, 北京 100190
}

\begin{abstract}
摘要: 有机太阳能电池具有成本低廉、质量轻、柔性可折叠以及可以大面积印刷等优点, 受到广泛关注。但与无机太阳 能电池相比, 其能量损失较高。在有机光伏分子中引入氟原子是一种有效提高器件性能的分子设计策略。本文从氟原子 特点出发, 总结了氟化给体、 $\pi$ 桥和受体单元对分子能级调控和形貌优化的作用, 阐明了氟原子降低能量损失的内在原 因; 并通过代表性分子设计实例, 简要阐述了氟化策略在高效聚合物给体材料、高效可溶性小分子给体材料以及高效非 富勒烯受体材料中的应用; 最后, 对氟化策略的应用进行了总结, 并展望了未来的研究方向。
\end{abstract}

关键词: 氟化; 能级; 形貌; 能量损失; 光电转换效率 中图分类号: 0646

\section{1 引言}

有机太阳能电池具有成本低廉、质量轻、柔性 可折叠以及可以大面积印刷等优点, 成为太阳能 光伏技术的研究热点 ${ }^{1-7}$ 。近年来, 通过新材料的 设计合成 ${ }^{8-12}$ 、器件结构的优化、活性层形貌的调 控以及工作机制的深入研究, 有机太阳能电池光 电转换效率 $(\mathrm{PCE})$ 得到了大幅度提升。其中文献报 导的基于可溶性有机光伏小分子的 PCE 已经超过 $11 \%{ }^{13,14}$, 而基于聚合物的 PCE 已经超过 $13 \%{ }^{8}$ 。 但是, 要实现有机太阳能电池的实际应用, 需要发 展更有效的分子设计策略, 开发更高效的光伏材 料, 实现器件性能的进一步提升。

2016年, Sinke教授15等基于Shockley-Queisser 模型的效率极限, 总结了不同光伏器件的最佳效 率与理论效率 $\left(\eta_{\mathrm{SQ}}\right)$ 的差距(图1)。无机半导体单晶 太阳能电池 (单晶硅, 砷化镓和磷化铟镓)的最高效 率可以到达理论效率的 $75 \%$ 以上, 多晶硅、薄膜以 及钙钛矿太阳能电池的最高效率可达理论效率的 50\%-75\%。然而, 基于有机聚合物和小分子的最 高效率只有理论效率的不到 $50 \%$ 。相比于无机太阳 能电池和钙钛矿, 有机太阳能电池效率与理论极 限的差别依然很大, 其中短路电流的损失达到
$20 \%$, 开路电压和填充因子的损失超过了 $50 \%$ (图 1) ${ }^{15}$ 。因此要设计出突破性的高效率光伏材料的关 键因素之一是如何从分子设计的角度提高光子利 用率和降低能量损失 ${ }^{15-18}$ 。

从吸收光谱和分子能级的角度考虑, 降低能 量损失需要在拓宽吸收光谱(即减小带隙)的同时 保持较高的开路电压。但是, 有机太阳能电池中, 保证开路电压降低带隙在很多情况下会导致外量 子效率(EQE)的降低。2011年, 埃因霍温科技大学 的 Janssen教授课题组 ${ }^{19}$, 基于吡咯并吡咯二酮 (DPP) 体系合成了一系列材料, 探讨其能量损失 $\left(E_{\mathrm{g}, \text { loss }}=\min \left(E_{\mathrm{g}, \text { acceptor }}, E_{\mathrm{g}, \mathrm{donor}}\right)-\mathrm{e} V_{\mathrm{oc}}\right.$; 其中 $E_{\mathrm{g}, \text { acceptor }}$ 和 $E_{\mathrm{g}, \mathrm{donor}}$ 指给体材料和受体材料的光学带隙) 与 $\mathrm{EQE}$ 的关系, 发现能量损失的增大与 $\mathrm{EQE}$ 提升呈 现线性关系。其中能量损失最低的只有 $0.65 \mathrm{eV}$, 但其 $\mathrm{EQE}$ 的最高值不到 $40 \%$, 因而其 PCE 只有 $4.7 \%$, 这主要是因为激子分离的驱动力过小 $\left(\Delta E_{\mathrm{LUMO}}\right.$, 给体和受体材料两者最低电子未占据轨 道能级之差) ${ }^{19}$ 。然而, 随着材料的不断丰富, 研 究者们也开发了一系列低能量损失高 $E Q E$ 值的材 料体系 $16,20,21$, 即降低能量损失和保证高 $\mathrm{EQE}$ 并不 矛盾。在较低的电荷分离驱动力下, 有机光伏材料

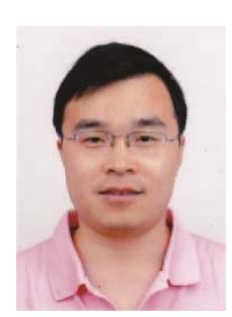

周二军, 研究员, 出生于 1980 年。 2007 年在中国科学院化学研究所获 得博士学位。2007 年至 2014 年先后 在日本科学技术振兴机构、日本东 京大学、日本理化学研究所从事科 学研究工作。2014 年 3 月被聘为国 家纳米科学中心研究员。主要从事有机光电材料的 设计合成及器件研究。

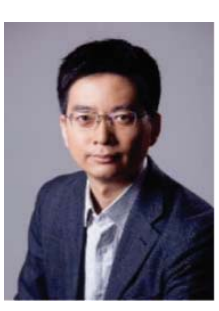

魏志祥, 研究员, 出生于1975年。 2003年在中国科学院化学研究所获 得博士学位。2003年至2005年, 先后 在德国马普胶体界面研究所和加拿 大多伦多大学从事博士后研究; 2006被聘为国家纳米科学中心研究 员。主要研究方向为有机光电材料的自组装和柔性 器件。 


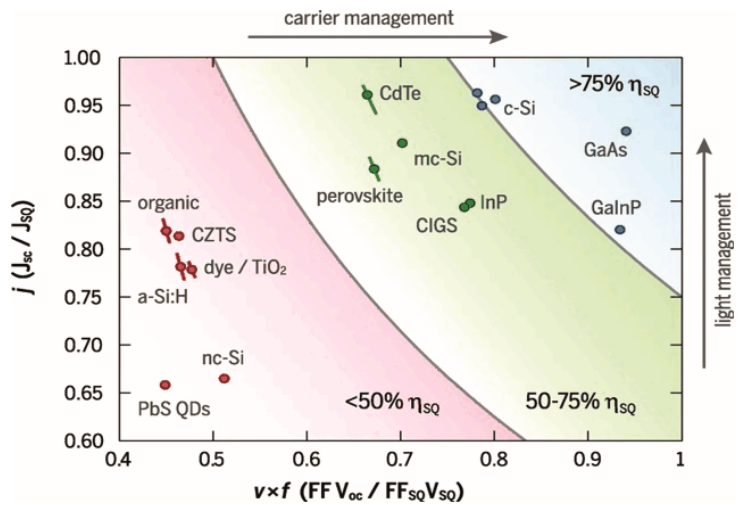

图1 各光伏材料器件性能的限制因素

Fig. 1 Limiting process in photovoltaic materials.

The figure is extracted from reference 15, copyright Science.

中的激子也可以有效的分离, 产生电荷。因此, 如 何在降低能量损失的同时提高光子的利用率是高 效光伏材料设计的关键问题。而氟化策略在调控 分子能级的同时可以实现活性层形貌的优化, 是 解决上述关键问题的有效途径之一 $22-24$ 。

因此，本文将重点介绍氟原子的特点和功能， 通过综述氟原子设计策略在高效聚合物给体材 料、高效可溶性小分子给体材料和非富勒烯受体 材料中的应用, 阐述氟化材料如何降低能量损失 的同时提高器件性能(包括高开路电压, 填充因子 和 $\mathrm{EQE})$, 为高效光伏材料的设计提供参考。

\section{2 氟原子作用}

氟原子是元素周期表中独特的元素, 它是电 负性最强的原子, 鲍林电负性为 4.0 , 远大于氢原
子。但是它的范德华半径很小 $(0.135 \mathrm{~nm})$, 只略比 氢原子大 $(0.12 \mathrm{~nm})^{22,24,25}$ 。因此, 相比于氰基和羰 基等取代物而言, 氟原子是最小的吸电子基团。在 组成有机光伏分子的典型给体、受体和 $\pi$ 桥单元中 $(\pi$ 桥大部分由噻吩, 苯环组成, 因此氟化 $\pi$ 桥包含 在氟化给体单元中), 均可以将不同位置的氢原子 替换为氟原子(图2)。

本部分将系统介绍氟原子在光伏材料能级调 控和形貌调控中的作用。

\section{1 氟原子调控能级}

氟原子作为最小的吸电子基团, 由于其强的电 负性，早期在有机光电材料的应用主要是在有机 场效应晶体管中。利用氟化可以降低最高电子占 据轨道(HOMO) 和最低电子未占据轨道( LUMO), 降低电子注入的势垒和提高空穴注入的势垒, 从 而实现从 $p$ 型半导体到双极性半导体再到 $n$ 型半导 体的调控，或者提高分子的 $n$ 型性能 ${ }^{26-28}$ 。2017年， 王佛松研究员课题组 ${ }^{29}$ 阐述了氟原子在调控能级 中的作用，并通过氟化实现了 $p$ 型材料到 $n$ 型材料 的转变。在有机光伏材料的设计中, 2009年, 加州 大学Yang 教授课题组 ${ }^{30}$ 将氟原子引入聚合物给体 材料PBDTTT-C的分子骨架中，用氟取代了受体 单元辛羰基取代的并噻吩(TT)的氢原子(对应的分 子为PBDTTT-CF)。相比于氟化前, 聚合物的 $\mathrm{HOMO}$ 能级从 $-5.12 \mathrm{eV}$ 降到了 $-5.22 \mathrm{eV}$, 开路电压 从 $0.70 \mathrm{~V}$ 提升到了 $0.76 \mathrm{~V}$, 器件性能提高到了 $7.7 \%$ 。2011年, 芝加哥大学俞陆平教授 31,32 基于聚 合物PBTF0, 在给体单元苯并二噻吩(BDT)的分子

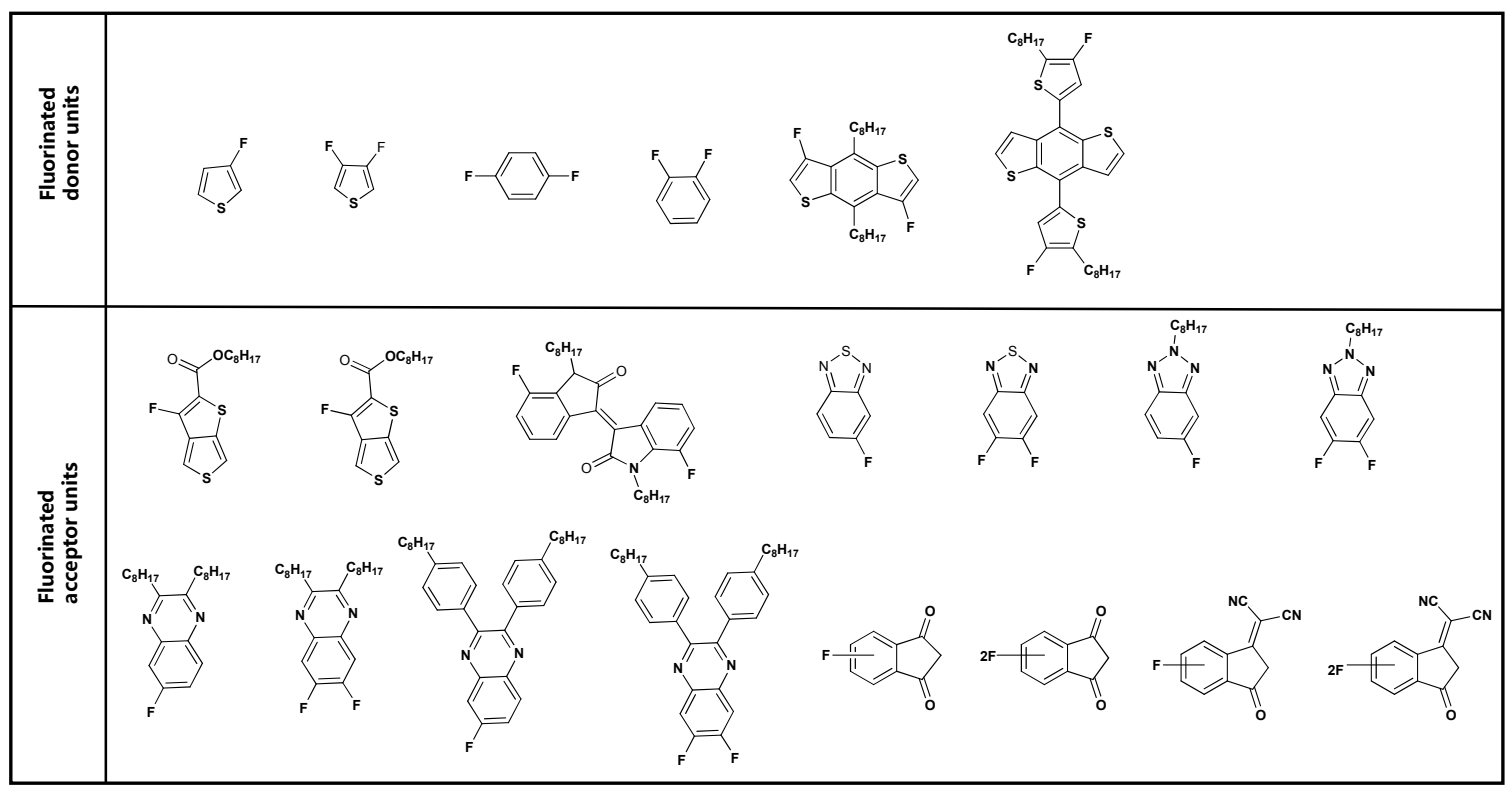

图 2 常见氟化的给体单元和受体单元

Fig. 2 Fluorinated donor units and acceptor units. 
骨架给体单元和受体单元 TT上分别或者同时引入 氟原子(PBTF1、PBTF2 和PBTF3), 其HOMO能级 从-4.94 eV降到了 $-5.48 \mathrm{eV}$, 但是开路电压并没有 如期望般增加。尤其对于PBTF1到PBTF3, 其开路 电压基本维持在 $0.70 \mathrm{~V}$, 且其器件性能从 $5 \%$ 降到 了 $2.7 \%$ 。这可能是因为处于分子骨架上的氟对电 子云密度调控能力过强, 从而降低了激子分离的 驱动力, 同时又对分子的有序堆积产生不利影响 导致的。2014年, 中国科学院化学研究所侯剑辉研 究员课题组 ${ }^{33}$ 基于 PBT-0F在噻吩取代的苯并二噻 吩(TBDT)的二维结构噻吩上(非分子骨架)和 TT上 引入氟原子 (PBT-1F、PBT-2F 和 PBT-3F), 其 HOMO 能级从 $-4.90 \mathrm{eV}$ 降低到了 $-5.20 \mathrm{eV}$, 其开路 电压从 $0.56 \mathrm{~V}$ 提升到了 $0.78 \mathrm{~V}$, 相应的器件性能从 $4.5 \%$ 提高到了 $8.6 \%$ 。2015年, 四川大学的彭强教 授课题组将氟取代的TBDT引入到小分子给体材 料 DR3TBDTT ${ }^{34}$, 设计并合成了分子 DR3TBDTTF。相比于氟化前, 其降低了HOMO能 级, 改进了开路电压和器件性能。综上所述, 氟原 子不论取代在给体还是受体单元, 聚合物还是小 分子均可以有效的降低HOMO和LUMO能级, 实 现整个分子能级下移。但是相对于取代在分子共 轭骨架上而言, 氟取代在二维结构上对分子能级 的调控更加精细可控。本章节所涉及的分子结构 见图3, 具体器件性能参数见表 1 。

\section{2 氟原子调控形貌}

由于氟原子的强电负性, 其可通过弱相互作 用力提高分子内平面性和分子间的相互作用力, 如 $F \cdots H 、 F \cdots S 、 F \cdots \pi$ 等 $22,24,35$ 。2012 年 Bazan 和 Heeger 5,36 教授课题组将氮原子引入以苯并噻二 唑为受体单元的小分子给体材料 $\left(\mathrm{DTS}\left(\mathrm{BTTh} \mathbf{h}_{2}\right)_{2}\right)$ 中合成分子 DTS(PTTh $\left.)_{2}\right)_{2}$, 使器件性能从 $0.18 \%$ 提 升到了 $6.7 \%$ 。这主要归因于分子聚集的有序性提 高, 使得其在膜中的吸收光谱红移幅度增大、分子 结晶性和迁移率大幅度提高。作者认为这种更有 序的聚集是由于氮的引入稳定了分子构象提高了 偶极矩方向的一致性。后来, 他们将氟原子取代 氮原子合成分子 DTS $\left(\text { FBTTh }{ }_{2}\right)_{2}$, 取得同样的效 果, 器件性能从 $6.7 \%$ 提升到了 $7.1 \% 37$ 。但是美 国西北大学 Ratner 教授 35 等计算了常见元素之间 的非共价键作用力对分子构象的影响, 发现在共 轭单元中, $F \cdots H$ 作为分子锁的作用并不如 $\mathrm{O} \cdots \mathrm{H}$ 和 $\mathrm{N} \cdots \mathrm{H}$ 。作者解释的是由于氟原子亲核能力不 强, 而且氟和 $\pi$ 电子作用力很强, 使得其被 $\pi$ 电 子所束缚, 导致它很难参与到分子内部的电子离 域, 所以其分子锁功能并不如 $\mathrm{N} \cdots \mathrm{H}$ 和 $\mathrm{O} \cdots \mathrm{H}$ 。相 比于 $\mathrm{O} \cdots \mathrm{S}$ 和 $\mathrm{N} \cdots \mathrm{S}, \mathrm{F} \cdots \mathrm{S}$ 的分子锁作用也要弱一 些。因此, 将氟引入分子骨架作为分子锁来稳定分 子构象的文献报导远不如氧和氮的多。

氟原子作为吸电子基团, 增大了分子的偶极, 加强了分子间的静电作用力从而利于分子间的聚 集 ${ }^{38}$ 。2011 年, 美国北卡罗莱纳大学 You 教授课

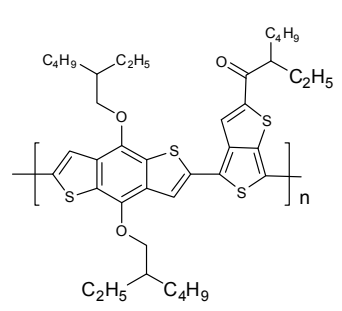

PBDTTT-C

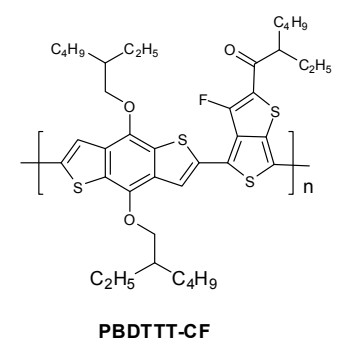

PBDTTT-CF

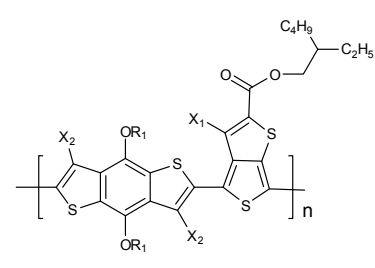

PBTF0: X1=H, X2=H, R1=n-octyl PBTF1: $X 1=F, X 2=H, R 1=2$-ethylhexyl PBTF2: $X 1=H, X 2=F$ R1=2-ethylhexyl PBTF3: X1=F, X2=F, R1=2-ethylhexyl

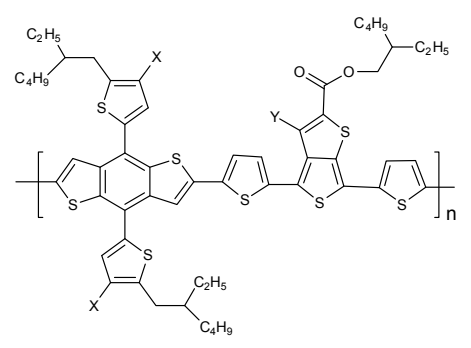

PBT -OF : $X=\mathrm{H}, \mathrm{Y=H}$ PBT -1F: $X=H, Y=F$ PBT -2F : $X=F, Y=H$
$P B T-3 F: X=F, Y=F$

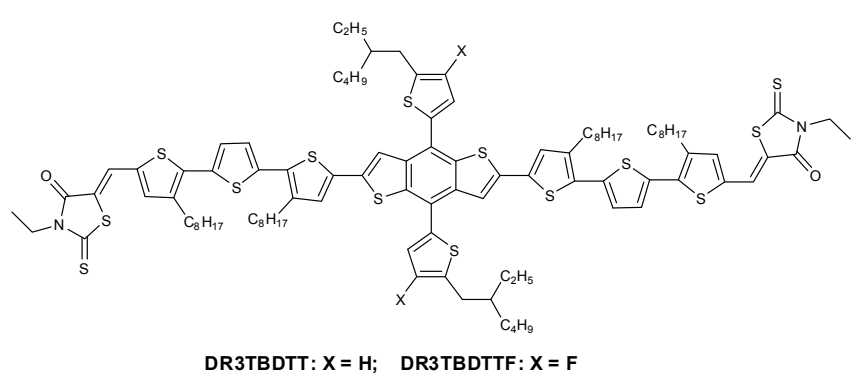

图 3 调控分子能级部分中的氟化分子结构

Fig. 3 Fluorinated molecular structures for tuning energy levels. 
表 1 基于氟的作用部分所涉及到的材料的具体器件性能参数

Table 1 Detailed parameters of the optimized device referred to fluorination effects.

\begin{tabular}{|c|c|c|c|c|c|c|c|c|}
\hline Device & $E_{\mathrm{HOMO}} / \mathrm{eV}$ & $E_{\text {LUMO }} / \mathrm{eV}$ & $V_{\mathrm{oc}} / \mathrm{V}$ & $J_{\mathrm{sc}} /\left(\mathrm{mA} \cdot \mathrm{cm}^{-2}\right)$ & $\mathrm{FF} / \%$ & $\mathrm{PCE} / \%$ & Hole mobility $/\left(\mathrm{cm}^{2} \cdot \mathrm{V}^{-1} \cdot \mathrm{s}^{-1}\right)$ & Ref. \\
\hline PBDTTT-C:PC ${ }_{71} \mathrm{BM}$ & -5.12 & -3.35 & 0.70 & 14.7 & 64.1 & 6.6 & $2.0 \times 10^{-4}$ & 30 \\
\hline PBDTTT-CF:PC ${ }_{71} \mathrm{BM}$ & -5.22 & -3.45 & 0.76 & 15.2 & 66.9 & 7.7 & $7.0 \times 10^{-4}$ & 30 \\
\hline PBTF0:PC ${ }_{71} \mathrm{BM}$ & -4.94 & -3.22 & 0.58 & 14.1 & 62.4 & 5.1 & $2.7 \times 10^{-4}$ & 32 \\
\hline PBTF1:PC ${ }_{71} \mathrm{BM}$ & -5.15 & -3.31 & 0.74 & 14.1 & 68.9 & 7.2 & $4.1 \times 10^{-4}$ & 32 \\
\hline PBTF2:PC ${ }_{71} \mathrm{BM}$ & -5.41 & -3.60 & 0.68 & 11.0 & 43.4 & 3.2 & $1.8 \times 10^{-4}$ & 32 \\
\hline PBTF3:PC ${ }_{71} \mathrm{BM}$ & -5.48 & -3.69 & 0.75 & 9.1 & 39.4 & 2.7 & $7.0 \times 10^{-5}$ & 32 \\
\hline PBT-0F:PC ${ }_{71} \mathrm{BM}$ & -4.90 & -3.10 & 0.56 & 12.2 & 66.7 & 4.5 & & 33 \\
\hline PBT-1F:PC ${ }_{71} \mathrm{BM}$ & -4.95 & -3.12 & 0.60 & 14.3 & 65.7 & 5.6 & & 33 \\
\hline PBT-2F:PC ${ }_{71} B M$ & -5.15 & -3.27 & 0.74 & 14.4 & 67.7 & 7.2 & & 33 \\
\hline PBT-3F:PC 71 BM & -5.20 & -3.30 & 0.78 & 15.2 & 72.4 & 8.6 & & 33 \\
\hline DR3TBDTT:PC ${ }_{71} \mathrm{BM}$ & -5.13 & -2.90 & 0.90 & 13.92 & 73.3 & 9.18 & $5.43 \times 10^{-4}$ & 34 \\
\hline DR3TBDTTF:PC ${ }_{71} \mathrm{BM}$ & -5.23 & -2.91 & 0.93 & 14.10 & 74.7 & 9.80 & $5.82 \times 10^{-4}$ & 34 \\
\hline $\operatorname{DTS}\left(\mathrm{BTTh}_{2}\right)_{2}: \mathrm{PC}_{71} \mathrm{BM}$ & -5.15 & -3.57 & 0.78 & 0.9 & 25.4 & 0.18 & $0.01^{\mathrm{a}}$ & 36 \\
\hline $\mathrm{DTS}\left(\mathrm{PTTh}_{2}\right)_{2}: \mathrm{PC}_{71} \mathrm{BM}$ & -5.22 & -3.72 & 0.78 & 14.4 & 59.3 & 6.70 & $0.20^{\mathrm{a}}$ & 36 \\
\hline $\mathrm{DTS}\left(\mathrm{FBTTh}_{2}\right)_{2}: \mathrm{PC}_{71} \mathrm{BM}$ & -5.12 & -3.34 & 0.81 & 12.8 & 68 & 7.0 & & 37 \\
\hline PBnDT-HTAZ:PC ${ }_{61} \mathrm{BM}$ & -5.29 & -2.87 & 0.71 & 11.28 & 54.5 & 4.36 & $2.94 \times 10^{-4}$ & 39 \\
\hline PBnDT-FTAZ:PC ${ }_{61} \mathrm{BM}$ & -5.36 & -3.05 & 0.79 & 12.45 & 72.2 & 7.10 & $1.03 \times 10^{-3}$ & 39 \\
\hline $\mathrm{F} 0: \mathrm{PC}_{71} \mathrm{BM}$ & -5.38 & & 0.731 & 11.27 & 46.6 & 3.84 & $1.70 \times 10^{-4}$ & 40 \\
\hline $\mathrm{F} 25: \mathrm{PC}_{71} \mathrm{BM}$ & -5.45 & & 0.742 & 12.27 & 54.3 & 4.94 & & 40 \\
\hline $\mathrm{F} 50: \mathrm{PC}_{71} \mathrm{BM}$ & -5.39 & & 0.764 & 12.44 & 62.3 & 5.92 & & 40 \\
\hline $\mathrm{F} 75: \mathrm{PC}_{71} \mathrm{BM}$ & -5.39 & & 0.780 & 12.21 & 64.9 & 6.18 & & 40 \\
\hline $\mathrm{F} 100: \mathrm{PC}_{71} \mathrm{BM}$ & -5.45 & & 0.797 & 12.75 & 70.6 & 7.17 & $1.20 \times 10-3$ & 40 \\
\hline PBnDT-DTBT :PC ${ }_{71} \mathrm{BM}$ & -5.42 & & 0.78 & 11.7 & 47.6 & 4.53 & $3.0 \times 10-4$ & 41 \\
\hline PBnDT-DTfBT:PC ${ }_{71} \mathrm{BM}$ & -5.48 & & 0.85 & 11.4 & 50.6 & 5.28 & $2.9 \times 10-4$ & 41 \\
\hline PBnDT-DTffBT:PC 71 BM & -5.53 & & 0.90 & 12.2 & 62.1 & 7.16 & $3.6 \times 10-4$ & 41 \\
\hline PDTQX:PC ${ }_{71} \mathrm{BM}$ & -5.48 & -3.66 & 0.79 & 4.67 & 42 & 1.56 & $6.0 \times 10^{-} 5$ & 42 \\
\hline PDFDTQX:PC ${ }_{71} \mathrm{BM}$ & -5.62 & -3.75 & 0.91 & 10.61 & 62 & 6.08 & $1.2 \times 10-3$ & 42 \\
\hline PNTz4T:PC 71 BM & -5.14 & -3.46 & 0.71 & 19.4 & 0.73 & 10.1 & $3.4 \times 10-3$ & 43 \\
\hline PNTz4TF2:PC 71 BM & -5.38 & -3.53 & 0.82 & 19.3 & 0.67 & 10.5 & $1.5 \times 10^{-3}$ & 43 \\
\hline PNTz4TF4:PC ${ }_{71} \mathrm{BM}$ & -5.49 & -3.56 & 0.93 & 10.5 & 0.66 & 6.5 & $1.8 \times 10-4$ & 43 \\
\hline
\end{tabular}

${ }^{a}$ hole mobility measured by organic field effect transistor methods, the others are measured by space charge limited current methods.

题组 ${ }^{39}$ 将氟引入苯并三唑(TAZ), 以 BDT 作为给 体单元的聚合物中 (PBnDTTAZ), 合成分子 PBnDTFTAZ。引入氟后, 聚合物的器件性能从 $4 \%$ 提升到了 $7 \%$, 这主要源于填充因子从 $50 \%$ 提升到 了 $70 \%$ 。为了深入探讨氟原子的引入如何影响填 充因子，他们合成了系列不同比例的氟取代的 $\mathrm{TAZ}$ (从 $0 、 0.25 、 0.5 、 0.75$ 到 1 ，对应聚合物分 别为 F0、F $25 、 F 50 、 F 75$ 和 F100) ${ }^{40}$, 发现随着氟 含量的增加, 其空穴迁移率依次提高, 从 $10^{-4}$ $\mathrm{cm}^{2} \cdot \mathrm{V}^{-1} \cdot \mathrm{s}^{-1}$ 逐渐提升到 $10^{-3} \mathrm{~cm}^{2} \cdot \mathrm{V}^{-1} \cdot \mathrm{s}^{-1}$ 。2012 年, 他们将一个/两个氟取代的苯并噻二唑(BT)作为聚 合物受体单元, 以 TBDT 作为给体单元合成聚合 物 PBnDT-DTBT、PBnDT-DTfBT 和 PBnDTDTffBT, 深入探讨氟原子个数对器件性能影响 ${ }^{41}$ 。 结果表明, 氟原子的引入不但提高了开路电压, 还
大幅度改进了电流和填充因子。作者通过测试不 同光强下的器件性能, 发现随着氟原子的增加, 双 分子复合降低; 通过模拟计算, 发现从基态到激发 态的偶极矩变化随着氟原子的增加而增加, 从而 降低了激子束缚能, 有利于激子分离。而这些表观 物理现象最终归因于氟原子对形貌和分子构象的 改进。共振软 X 射线散射(R-SoXs)结果证明氟化 增强了材料结晶相的相纯度, 掠入射 $\mathrm{X}$ 散射结果 (GIWAXS)证明氟化增加了材料堆积的有序长度。 但是在这个体系中，氟的引入并没有增加材料在 纯相的迁移率。在混合相里由于氟的引入使得给 体材料的聚集方式更趋近于 face-on 排列，从而提 高了迁移率。韩国的 Kim 教授课题组 ${ }^{42}$ 也将氟引 入了受体单元喹喔啉中 $(\mathrm{QX})$ ，合成聚合物 PDFDTQX。氟的引入同样提高了其迁移率, 改善 
了填充因子。

在给体单元噻吩上引入氟原子同样也可以调 控形貌。2016 年, 日本 Takimiya 教授课题组 ${ }^{43}$ 探 讨了 $\pi$ 桥上氟的饱和度对器件性能的影响, 合成 分子 PNTz4T、PNTz4TF2 和 PNTz4TF4。当引入 两个氟原子时, 其器件性能最好(从 $10.1 \%$ 提升到 $10.5 \%$ ), 这主要归因于其 HOMO 能级的降低, 开 路电压的增加 (从 $0.71 \mathrm{~V}$ 增加到 $0.82 \mathrm{~V}$ )。当引入 四个氟原子之后由于分子间聚集作用力太强, 所 以分子堆积没有固定的取向, 从而降低了其迁移 率, 导致其无法通过增加膜厚来增加吸收的光子, 使得电流和器件性能低(6.5\%)。另外, 氟化 $\pi$ 桥可 以有效降低电荷复合、提高电流, 在聚合物受体材 料的工作中同样也有报导 44 。

综上所述, 不论氟原子取代在给体单元还是受 体单元均对形貌产生影响, 具体表现为增加分子堆 积的有序长度、提高相纯度、降低分子在电荷传输 方向上堆积距离、在某些体系甚至可以诱导face-on 的聚集。这些对形貌的调控作用大部分是有利的, 比如提高了电荷传输能力、降低了电荷复合, 从而 提升了电流和填充因子, 改进器件性能。同时, 在 引入氟原子时需要考虑其饱和度的问题, 如果氟引 入过多, 分子间作用力太强, 反而使得分子堆积无 序。另外, 在聚合物中, 单氟的引入由于其结构的 不对称性容易降低分子的规整性, 从而导致分子的 无序度增加, 降低器件性能。本章节所涉及的分子 结构见图4, 具体参数见表1。

\section{3 氟化策略在高效有机光伏材料中的应用 举例}

在上一节系统总结氟原子对分子能级和形貌 调控的基础上, 本节将重点通过代表性实例介绍
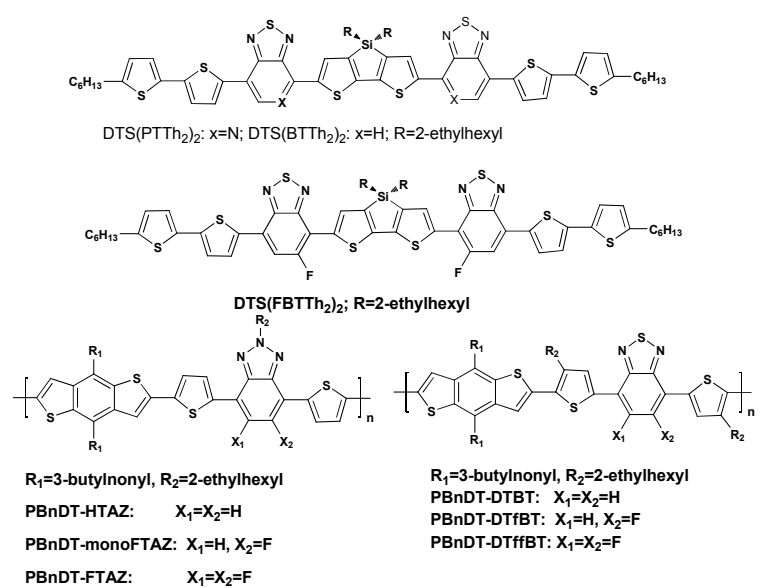

图 4 调控形貌部分中的氟化分子结构
氟化策略在高效光伏材料体系中的应用, 具体包 括三个方面: 高效聚合物给体材料、高效可溶性小 分子给体材料以及高效非富勒烯受体材料。

\section{1 高效聚合物中的应用}

2016 年, 中科院化学研究所侯剑辉研究员课 题组以 ITIC 作为受体材料, 基于 TBDT 为给体单 元、喹喔啉为受体单元的聚合物给体材料 (PBQ$\mathbf{0 F}$ ), 探讨了氟化聚合物的给体单元和受体单元对 分子光电性质和器件性能的影响(图 5) ${ }^{21}$ 。两个氟 原子引入受体单元喹喔啉对应的聚合物为 PBQ$\mathbf{Q F}$, 再将两个氟原子引入给体单元 TBDT 对应 的聚合物为 PBQ-4F (图 5a)。随着氟原子的引入, 聚合物的 $\mathrm{HOMO}$ 能级从 $-5.18 \mathrm{eV}$ 降到 $-5.34 \mathrm{eV}$ 再 到 $-5.49 \mathrm{eV}$, 其相应的开路电压从 $0.69 \mathrm{~V}$ 提升到 $0.83 \mathrm{~V}$ 再到 $0.95 \mathrm{~V}$ 。同时其填充因子从 $59.9 \%$ 提升 到 $63 \%$ 再到 $66 \%$, 使得其器件性能从 $6.68 \%$ 改进 到 $8.9 \%$ 再到 $11.3 \%$ (图 5b-c)。填充因子的提高主 要源于氟原子的引入提升了材料结晶相的相纯度 (图 5d)。能量损失是由给体材料和受体材料间较 小的光学带隙减去其开路电压对应的能量。相比 于给体材料, 受体材料 ITIC 具有更小的光学带隙 $(1.57 \mathrm{eV})$ 。因此 PBQ-0F、PBQ-QF 和 $\mathbf{P B Q}-\mathbf{4 F}$ 对 应的能量损失分别为 $0.88 、 0.74$ 和 $0.62 \mathrm{eV}$ 。即随 着氟原子的增加, 其能量损失大幅度降低, 其极限 效率也大幅度提升。同时, 器件性能的光子利用率 也提高了(其 $\mathrm{EQE}$ 的最高值从 $77 \%$ 提升到 $80 \%$ 和 $81 \%$, 其填充因子和电流均有提升。因此, 对于 这个体系而言, 氟化策略成功地在降低能量损失 的同时提高了光子利用率, 改进了器件性能。

\section{2 可溶性小分子给体材料的应用}

2016 年, 魏志祥研究员课题组设计了一种新 型的 $\pi$ 桥, 并以 TBDT 作为给体单元, 以狮二酮
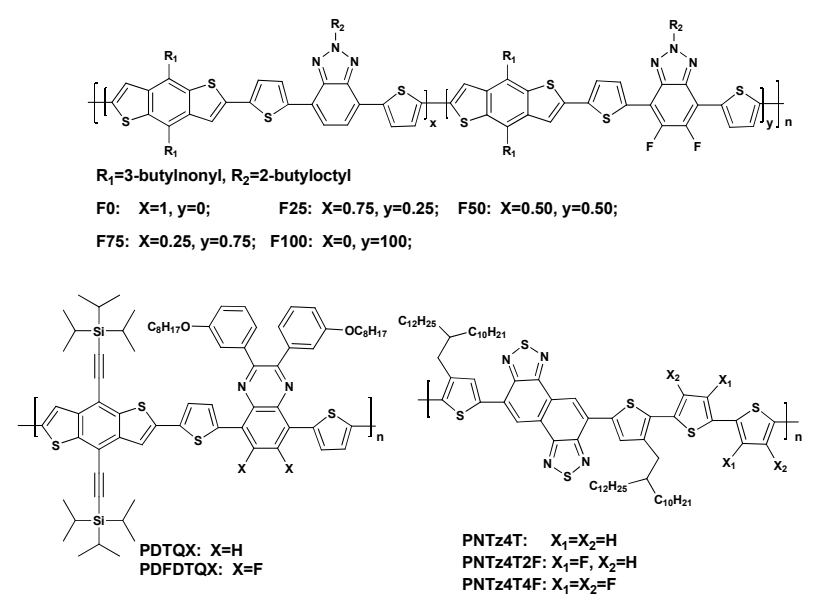

Fig. 4 Molecular structures for tuning morphology. 
a)

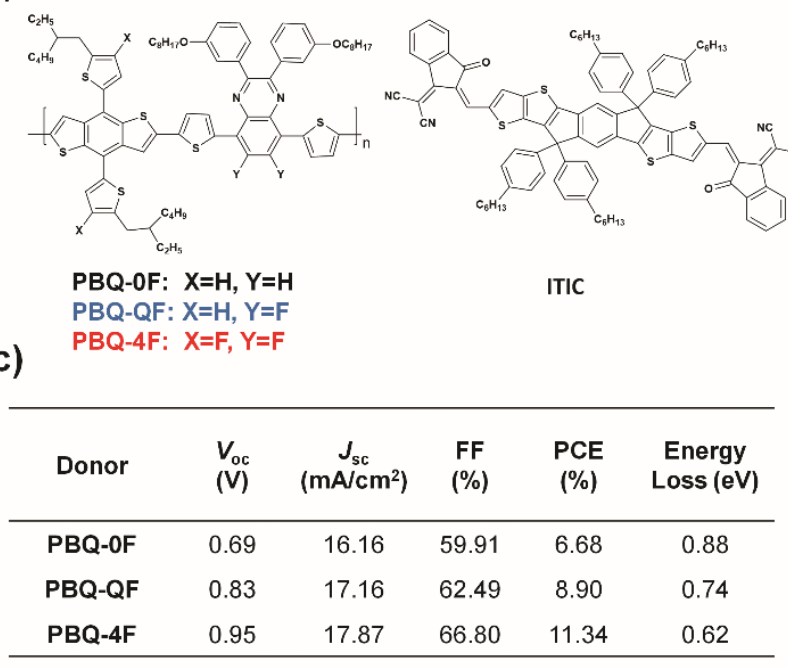

b)

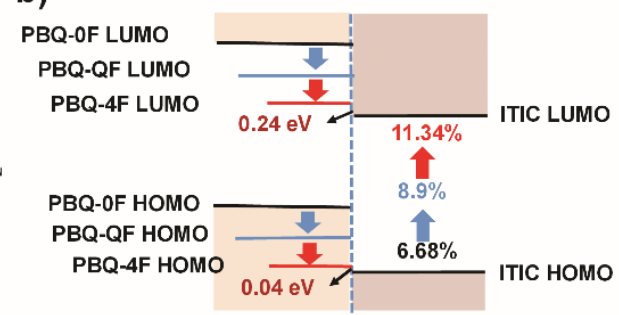

d)

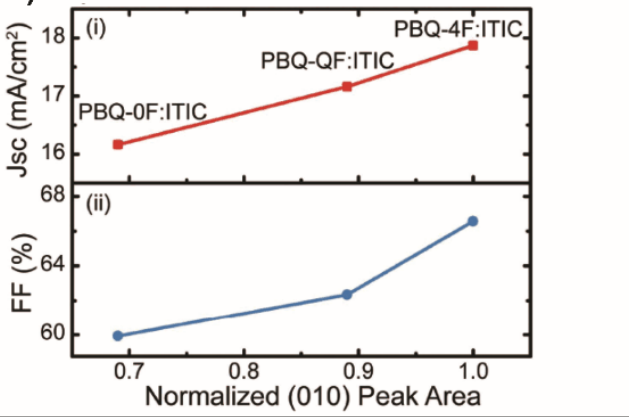

图 5 氟化在高效聚合物给体材料的应用的实例

Fig. 5 Detailed information for fluorination application in efficient polymers.

a) molecular structure b) energy levels c) parameters of device performance d)the positive relationship between device parameters (FF and $J_{\mathrm{sc}}$ ) with the crystallinity in $\pi-\pi$ stacking direction. The figure is extracted from reference 21 , copyright Wiley online library.

为受体端基，合成分子 BTID-0F (图 6) ${ }^{13,45}$ 。基于 $\mathrm{PC}_{71} \mathrm{BM}$ 为受体材料, BTID-0F 的正向器件性能达 到了 $10.1 \%$ 。但是相对而言性能更稳定、寿命更长 的反向器件, 其 PCE 只有 $8.3 \%$ 。这主要是因为正
向器件结构和反向器件结构的载流子传输方向是 相反的, 因而对活性层在垂直方向上的相形貌要 求不一致。而正是这种垂直相形貌的要求不一致 导致了正反向器件性能之间的差异。通过氟化茆

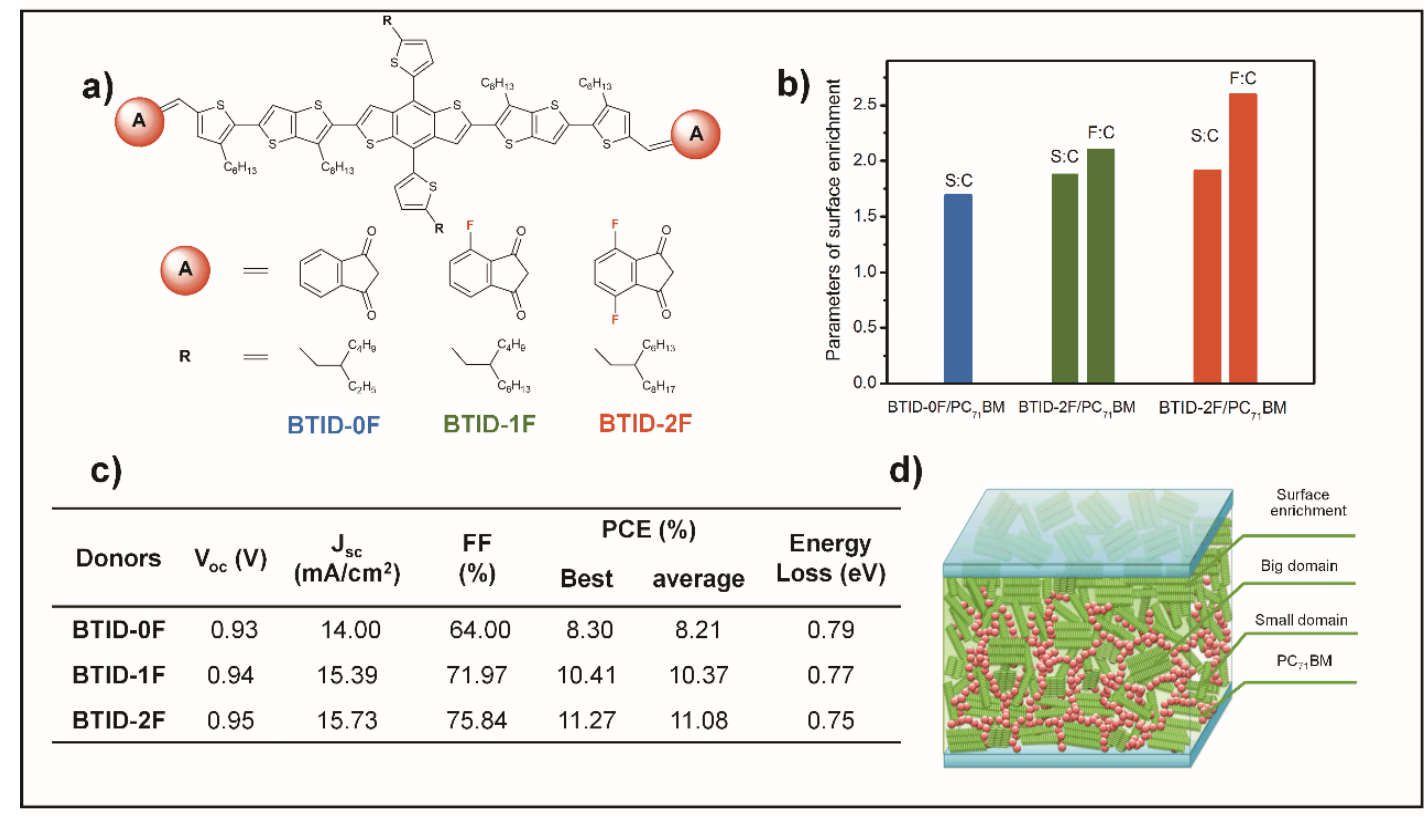

图 6 氟化在可溶性小分子给体材料应用的实例

Fig. 6 Detailed information for fluorination application in efficient solution-processable small molecules.

a) molecular structure, b) fluorination effects on surface enrichment, c) device performances, d) ideal morphology of active layer; the figure is extracted from reference 13, copyright Nature. 
二酮受体端基可以轻微降低给体材料的表面张 力, 调控垂直方向的相形貌, 使得其更适应于反向 器件结构。在受体端基分别引入一个氟和两个氟, 对应的分子为 BTID-1F 和 BTID-2F (图 6a)。

接触角的结果表明, 氟化受体端基确实增大 了接触角降低了表面张力, 同时氟化也增加了给 体小分子和受体材料 $\mathrm{PC}_{71} \mathrm{BM}$ 表面张力的差异性, 即降低了两种材料的共混性, 增大了相分离的驱 动力。GIWAXS 测试结果表明, 氟化受体端基增 强了给体材料在电荷传输方向的有序堆积, 并且 加入受体材料 $\mathrm{PC}_{71} \mathrm{BM}$ 后, 对其有序堆积的影响 较小。R-SoXs 的结果表明, 氟化受体端基增加了 给体材料结晶相的相纯度。并且联合原子力显微 镜和透视电子显微镜的结果表明, 在活性层中, 同 时存在利于电荷传输的尺度大的颗粒 (大约 100 $\mathrm{nm}$ ) 和利于电荷产生的尺度小的颗粒 (大约 20 $\mathrm{nm}$ ), 这种多级次形貌有利于平衡电荷分离和传 输。垂直方向上, 氟化受体端基提高了表面富集程 度, 尤其是 BTID-2F/PC ${ }_{71} \mathrm{BM}$, 在上表面完全富集 了一层给体材料, 形成电子阻挡层(图 6b); 并且在 垂直方向上形成给体材料递增的单向性分布, 进 而改进电荷收集和电荷输运, 降低了电荷复合。因 此基于 BTID-2F 的器件性能达到了 11.3\% (图 6c)。 针对水平和垂直相形貌, 提出了反向器件的理想 活性层形貌, 即多级次相尺度分布和表面富集给 体材料的单向性分布(图 $6 \mathrm{~d}$ )。基于 $\mathrm{PC}_{71} \mathrm{BM}$ 为受 体, BTID-0F、BTID-1F 和 BTID-2F 的能量损失 分别为 $0.79,0.77$ 和 $0.75 \mathrm{eV}$ 。因为从紫外光电子 能谱结果来看, 氟化受体端基对 HOMO 能级只有 微调, 因此这三者的能量损失相差并不大。器件性 能的改进主要归因于氟化端基对活性层水平和垂 直相形貌的调控。

另外, 对于 BTID-2F, 由于其垂直相形貌利 于反向器件结构, 因此其正向器件性能比反向低。 通过提高其基底甩膜温度, 弱化垂直相分布, 可以 实现开路电压损失的降低, 改进器件性能 45 。

\section{3 高效非富勒烯受体的应用}

相比于富勒烯而言, 非富勒烯受体材料可以 大幅度红移吸收光谱和增加吸光系数, 并且其光 电性能容易通过化学修饰调控, 受到研究者们的 青睐。其中最为成功的是大稠环结构为给体单元 的, 二氰基取代的茚二酮作为受体端基的 $\mathrm{A}$ (acceptor)-D (donor)-A (acceptor)型小分子。2017 年, 中国科学院化学研究所的侯剑辉研究员和北 京大学的占肖卫教授先后氟化了二氰基取代的狮 二酮的受体端基 ${ }^{8,46}$, 均取得不错的器件性能。在
本小节中, 我们具体以占肖卫教授课题组的 INIC 系列工作为例进行介绍。以 IBDT 为给体单元, 以 不同的氟化位置或氟化饱和度的二氰基取代的狮 二酮作为受体端基, 对应的分子分别为 INIC、

INIC1、INIC2 和 INIC3 (图 7a)。基于聚合物 FTAZ 作为给体材料, 这四个受体材料相应的器件分别 获得 $7.7 \%$ 、 $10.1 \% 、 10.8 \%$ 和 $11.5 \%$ 的光电转换效 率。随着氟原子个数的增加器件性能的提高主要 来源于电流和填充因子的提高。氟化受体依然降 低了 HOMO 和 LUMO 能级, 尤其是 LUMO 能级 从 $-3.88 \mathrm{eV}$ 降到了 $-4.03 \mathrm{eV}$ 。由于开路电压一般情 况下与 $\left(E_{\mathrm{LUMO}}\right.$ acceptor $\left.-E_{\mathrm{HOMO} \text { donor }}\right)$ 成正相关, 随着 受体材料的 LUMO 的下降, 其开路电压下降。因 此基于 FTAZ 作为给体材料, 随着氟原子个数的 增加, 开路电压从 $0.953 \mathrm{~V}$ 降到了 $0.857 \mathrm{~V}$ (图 $7 \mathrm{~b}-\mathrm{c}$ )。氟化 INIC 增加了受体单元强度, 同时也红 移了吸收光谱, 降低了带隙。降低的开路电压恰好 跟减小的带隙所补偿, 所以基于 INIC、INIC1、 INIC2 和 INIC3 这四者的能量损失基本没变化, 对应的值分别为 $0.61 、 0.63 、 0.62$ 和 $0.62 \mathrm{eV}$ 。针 对电流和填充因子提高的原因, 作者认为氟化不 仅诱导了 face-on 排列(图 7d), 而且增强了分子结 晶性能, 同时也使得给受体材料排列的更紧密; 在 提高电子迁移率同时也增加了电荷产生的界面, 从而降低了复合。因此, 其外量子效率的最高值从 大约 $60 \%$ 提升到了大约 $80 \%$, 填充因子从 $57.9 \%$ 提升到 $67.4 \%$ 。但部分原因也可能是激子分离的驱 动力增加 $(\triangle \mathrm{HOMO}$, 给受体的最高电子占据轨道 能级差)。

值得一提的是, 中国科学院化学研究所侯剑 辉研究员课题组和北京交通大学张福俊教授课题 组 47,48 先后将吸收光谱在近红外的 IEICO-4F(氟化 的非富勒烯受体材料)应用到三元器件中, 提高了 电流, 获得了不错的器件性能。

在这一章节里面, 我们简要的通过实例探讨 了氟化策略对高效聚合物给体材料、高效可溶性 小分子给体材料以及高效非富勒烯受体材料的光 电性质和器件性能的改进。这些实例有代表性, 但 并不全面。这三个实例当中, 氟化聚合物大幅度降 低能量损失的同时提高了光子利用率。氟化可溶 性小分子的实例重点是调控垂直相分布和活性层 水平形貌从而减少了电荷复合, 改进反向器件性 能。氟化非富勒烯受体材料降低了 LUMO 能级, 因而其开路电压降低, 但是氟化提高了电子迁移 率和光子利用率, 进而改进了器件性能。因此在氟 化非富勒烯受体的时候对能级需要特别注意, 不 


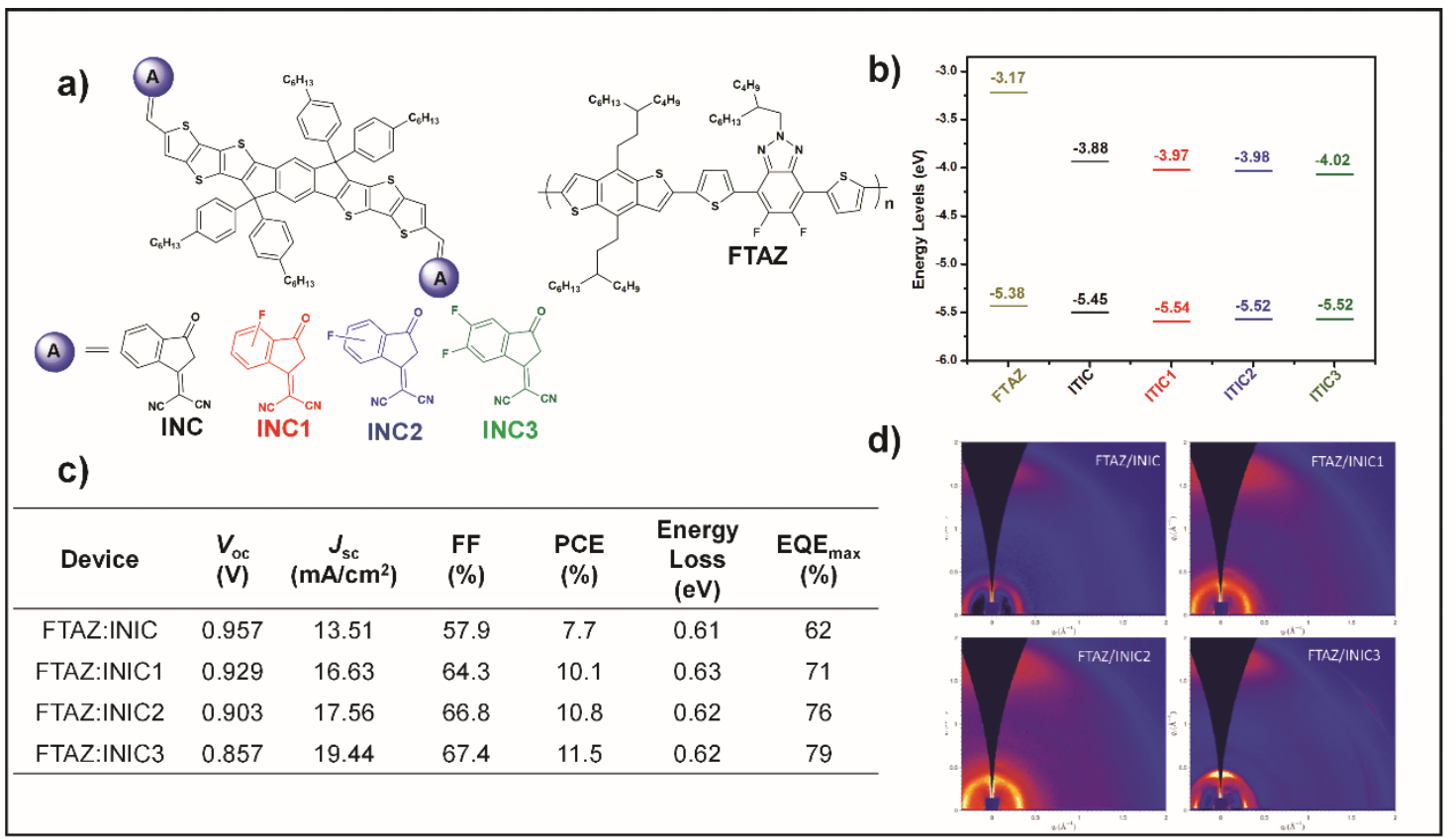

图 7 氟化在非富勒烯受体材料应用的实例

Fig. 7 Detailed information for fluorination application in non-fullerene materials.

a) molecular structure, b)energy levels, c)device performances, d) fluorination effects on microstructures.

The figure is extracted from reference 46, copyright American Chemical Society.

要将 LUMO 拉的太低, 导致开路电压大幅降低。

\section{4 总结与展望}

本文从氟原子特点出发, 从下移分子能级和 调控形貌两个方面, 综述了氟化在有机光伏材料 设计中的独特作用。通过典型实例, 进一步阐述了 氟化作用在高效光伏材料设计中的应用。氟化材 料通过下移给体材料的能级提高了开路电压, 通 过增强材料的结晶性能和相纯度等形貌调控提高 光子利用率, 改进了器件性能。值得注意的是, 氟 化策略在很多体系均有效, 但不是所有的体系都 适合氟化。比如下列两类材料就不适合氟化:一类 是材料的结晶性很好, 给体材料和受体材料的共 混性和相分离尺度合适的光伏材料; 另一类是能 级不适合再下移的光伏材料, 尤其是部分非富勒 烯受体材料, 氟化会拉低LUMO能级, 降低开路电 压。如果体系适合氟化, 还需要考虑氟化位置和饱 和度。否则氟化过度, 同样会出现相分离尺度过大 或者能级不匹配, 导致电荷产生效率不高器件性 能大幅度下降或者器件不工作。

窄带隙低能量损失的非富勒烯受体体系的成 功, 使得有机太阳能电池效率实现了从 $11 \%$ 到 $13 \%$ 的突破。该类材料在拓宽吸收光谱的同时, 在较低 的驱动力下激子就能进行有效的分离, 实现了开 路电压和光子利用率的同时提高, 提高了有机太
阳能电池的效率。同时, 为有机太阳能电池机理研 究和形貌调控提出了新的挑战和要求。具体包括: （1）窄带隙活性层材料的设计 (大约 $1.4 \mathrm{eV}$ ): 不仅 包括窄带隙非富勒烯和宽带隙给体材料，也包括 宽带系非富勒烯或者富勒烯受体材料和窄带隙给 体材料。(2) 低驱动力下激子有效分离的机理研 究, 并能反馈到分子设计原则上。虽然已经发现多 个低驱动力激子有效分离的体系，但是对于大多 数体系而言, 这种低驱动力下电荷分离并不是很 有效。对于如何将分子材料与低驱动力工作机制 关联起来, 并进一步指导分子的设计合成具有一 定挑战。(3) 形貌的调控。基于富勒烯体系的材料 对于形貌调控基本成熟, 但是, 基于非富勒烯体 系, 除了给体/受体材料的相纯度和结晶行为, 给 体/受体材料的共混性需要进一步深入研究。特别 是, 相比于富勒烯电子三维传输而言, 非富勒烯是 二维传输, 因此对于形貌的要求更苛刻。基于窄 带隙非富勒烯体系, 氟化策略提供了一种进一步 降低能量损失和调控精细形貌的手段, 在未来 更高效的材料设计中将成为一种有效的分子设计 方法。

\section{References}

(1) Liu, Y.; Zhao, J.; Li, Z.; Mu, C.; Ma, W.; Hu, H.; Jiang, K.; Lin, H.; Ade, H.; Yan, H. Nat. Commun. 2014, 5, 5293. 
doi: $10.1038 /$ ncomms6293

(2) Li, Y. Acc. Chem. Res. 2012, 45 (5), 723. doi: 10.1021/ar2002446

(3) Coughlin, J. E.; Henson, Z. B.; Welch, G. C.; Bazan, G. C. Acc. Chem. Res. 2014, 47 (1), 257. doi: 10.1021/ar400136b

(4) Chen, J.; Cao, Y. Acc. Chem. Res. 2009, 42 (11), 1709. doi: $10.1021 / \operatorname{ar} 900061 \mathrm{z}$

(5) Sun, Y.; Welch, G. C.; Leong, W. L.; Takacs, C. J.; Bazan, G. C.; Heeger, A. J. Nat. Mater. 2012, 11 (1), 44. doi: 10.1038/nmat3160

(6) Roncali, J. Acc. Chem. Res. 2009, 42 (11), 1719. doi: $10.1021 / \operatorname{ar} 900041 \mathrm{~b}$

(7) Zhang, J.; Deng, D.; He, C.; He, Y.; Zhang, M.; Zhang, Z. G.; Zhang, Z.; Li, Y. Chem. Mater. 2010, 23 (3), 817. doi: 10.1021/cm102077j

(8) Zhao, W.; Li, S.; Yao, H.; Zhang, S.; Zhang, Y.; Yang, B.; Hou, J. J. Am. Chem. Soc. 2017, 139 (21), 7148. doi: 10.1021/jacs.7b02677

(9) Yang, L.; Zhang, S.; He, C.; Zhang, J.; Yao, H.; Yang, Y.; Zhang, Y.; Zhao, W.; Hou, J. J. Am. Chem. Soc. 2017, 139 (5), 1958. doi: $10.1021 /$ jacs.6b11612

(10) Lin, Y.; Wang, J.; Zhang, Z. G.; Bai, H.; Li, Y.; Zhu, D.; Zhan, X. Adv. Mater. 2015, 27 (7), 1170. doi: 10.1002/adma.201404317

(11) Yang, Y.; Zhang, Z. G.; Bin, H.; Chen, S.; Gao, L.; Xue, L.; Yang, C.; Li, Y. J. Am. Chem. Soc. 2016, 138 (45), 15011. doi: $10.1021 /$ jacs.6b09110

(12) Hou, J.; Inganäs, O.; Friend, R. H.; Gao, F. Nat. Mater. 2018, 17, 119. doi: $10.1038 /$ nmat5063

(13) Deng, D.; Zhang, Y.; Zhang, J.; Wang, Z.; Zhu, L.; Fang, J.; Xia, B.; Wang, Z.; Lu, K.; Ma, W.; et al. Nat. Commun. 2016, 7, 13740. doi: $10.1038 /$ ncomms 13740

(14) Wan, J.; Xu, X.; Zhang, G.; Li, Y.; Feng, K.; Peng, Q. Energy Envirn. Sci. 2017, 10 (8), 1739. doi: 10.1039/C7EE00805H

(15) Polman, A.; Knight, M.; Garnett, E. C.; Ehrler, B.; Sinke, W. C. Science 2016, 352 (6283). doi: 10.1126/science.aad4424

(16) Kawashima, K.; Tamai, Y.; Ohkita, H.; Osaka, I.; Takimiya, K. Nat. Commun. 2015, 6, 10085. doi: 10.1038/ncomms 10085

(17) Zhang, J.; Zhu, L.; Wei, Z. Small Methods 2017, 1 (12), 1700258. doi: $10.1002 /$ smtd.201700258

(18) Liu, J.; Chen, S.; Qian, D.; Gautam, B.; Yang, G.; Zhao, J.; Bergqvist, J.; Zhang, F.; Ma, W.; Ade, H.; et al. Nat. Energy 2016, 1, 16089. doi: 10.1038/nenergy.2016.89

(19) Li, W.; Roelofs, W. S. C.; Wienk, M. M.; Janssen, R. A. J. J. Am. Chem. Soc. 2012, 134 (33), 13787. doi: 10.1021/ja305358z

(20) Bin, H.; Zhang, Z. G.; Gao, L.; Chen, S.; Zhong, L.; Xue, L.; Yang, C.; Li, Y. J. Am. Chem. Soc. 2016, 138 (13), 4657. doi: $10.1021 /$ jacs.6b01744

(21) Zheng, Z.; Awartani, O. M.; Gautam, B.; Liu, D.; Qin, Y.; Li, W.; Bataller, A.; Gundogdu, K.; Ade, H.; Hou, J. Adv. Mater. 2017, 29 (5), 1604241. doi: 10.1002/adma.201604241
(22) Xu, X. P.; Li, Y.; Luo, M. M.; Peng, Q. Chin. Chem. Lett. 2016, 27 (8), 1241. doi: 10.1016/j.cclet.2016.05.006

(23) Meyer, F. Prog. Polym. Sci. 2015, 47, 70. doi: 10.1016/j.progpolymsci.2015.04.007

(24) Zhang, Q.; Kelly, M. A.; Bauer, N.; You, W. Acc. Chem. Res. 2017, 50 (9), 2401. doi: 10.1021/acs.accounts.7b00326

(25) Kohlhepp, S. V.; Gulder, T. Chem. Soc. Rev. 2016, 45 (22), 6270. doi: 10.1039/C6CS00361C

(26) Zaumseil, J.; Sirringhaus, H. Chem. Rev. 2007, 107 (4), 1296. doi: $10.1021 / \operatorname{cr} 0501543$

(27) Delgado, M. C. R.; Pigg, K. R.; da Silva Filho, D. A.; Gruhn, N. E.; Sakamoto, Y.; Suzuki, T.; Osuna, R. M.; Casado, J.; Hernández, V.; Navarrete, J. T. L.; et al. J. Am. Chem. Soc. 2009, 131 (4), 1502. doi: $10.1021 / \mathrm{ja} 807528 \mathrm{w}$

(28) Tang, M. L.; Reichardt, A. D.; Wei, P.; Bao, Z. J. Am. Chem. Soc. 2009, 131 (14), 5264. doi: 10.1021/ja809659b

(29) Gao, Y.; Deng, Y.; Tian, H.; Zhang, J.; Yan, D.; Geng, Y.; Wang, F. Adv. Mater. 2017, 29 (13), 1606217. doi: 10.1002/adma.201606217

(30) Chen, H. Y.; Hou, J.; Zhang, S.; Liang, Y.; Yang, G.; Yang, Y.; Yu, L.; Wu, Y.; Li, G. Nat. Photon. 2009, 3, 649. doi: $10.1038 /$ nphoton.2009.192

(31) Rolczynski, B. S.; Szarko, J. M.; Son, H. J.; Liang, Y.; Yu, L.; Chen, L. X. J. Am. Chem. Soc. 2012, 134 (9), 4142. doi: 10.1021/ja209003y.

(32) Son, H. J.; Wang, W.; Xu, T.; Liang, Y.; Wu, Y.; Li, G.; Yu, L. J. Am. Chem. Soc. 2011, 133 (6), 1885. doi: 10.1021/ja108601g

(33) Zhang, M.; Guo, X.; Zhang, S.; Hou, J. Adv. Mater. 2014, 26 (7), 1118. doi: 10.1002/adma.201304427

(34) Wang, Z.; Xu, X.; Li, Z.; Feng, K.; Li, K.; Li, Y.; Peng, Q. Adv. Electron Mater. 2016, 2 (6), 1600061. doi: 10.1002/aelm.201600061

(35) Jackson, N. E.; Savoie, B. M.; Kohlstedt, K. L.; Olvera de la Cruz, M.; Schatz, G. C.; Chen, L. X.; Ratner, M. A. J. Am. Chem. Soc. 2013, 135 (28), doi: 10475. 10.1021/ja403667s

(36) Takacs, C. J.; Sun, Y.; Welch, G. C.; Perez, L. A.; Liu, X.; Wen, W.; Bazan, G. C.; Heeger, A. J. J. Am. Chem. Soc. 2012, 134 (40), 16597. doi: $10.1021 / \mathrm{ja} 3050713$

(37) van der Poll, T. S.; Love, J. A.; Nguyen, T. Q.; Bazan, G. C. Adv. Mater. 2012, 24 (27), 3646. doi: 10.1002/adma.201201127

(38) Ying, L.; Hsu, B. B. Y.; Zhan, H.; Welch, G. C.; Zalar, P.; Perez, L. A.; Kramer, E. J.; Nguyen, T. Q.; Heeger, A. J.; Wong, W. Y.; et al. J. Am. Chem. Soc. 2011, 133 (46), 18538. doi: 10.1021/ja207543g

(39) Price, S. C.; Stuart, A. C.; Yang, L.; Zhou, H.; You, W. J. Am. Chem. Soc. 2011, 133 (12), 4625. doi: 10.1021/ja1112595

(40) Li, W.; Albrecht, S.; Yang, L.; Roland, S.; Tumbleston, J. R.; McAfee, T.; Yan, L.; Kelly, M. A.; Ade, H.; Neher, D.; et al. J. Am. Chem. Soc. 2014, 136 (44), 15566. doi: 10.1021/ja5067724 
(41) Stuart, A. C.; Tumbleston, J. R.; Zhou, H.; Li, W.; Liu, S.; Ade, H.; You, W. J. Am. Chem. Soc. 2013, 135 (5), 1806. doi: $10.1021 / \mathrm{ja} 309289 \mathrm{u}$

(42) Kim, J. H.; Song, C. E.; Kim, H. U.; Grimsdale, A. C.; Moon, S. J.; Shin, W. S.; Choi, S. K.; Hwang, D. H. Chem. Mater. 2013, 25 (13), 2722. doi: $10.1021 / \mathrm{cm} 401527 \mathrm{~b}$

(43) Kawashima, K.; Fukuhara, T.; Suda, Y.; Suzuki, Y.; Koganezawa, T.; Yoshida, H.; Ohkita, H.; Osaka, I.; Takimiya, K. J. Am. Chem. Soc. 2016, 138 (32), 10265. doi: 10.1021/jacs.6b05418

(44) Uddin, M. A.; Kim, Y.; Younts, R.; Lee, W.; Gautam, B.; Choi, J.; Wang, C.; Gundogdu, K.; Kim, B. J.; Woo, H. Y. Macromolecules 2016, 49 (17), 6374. doi: 10.1021/acs.macromol.6b01414
(45) Zhang, Y.; Deng, D.; Wang, Z.; Wang, Y.; Zhang, J.; Fang, J.; Yang, Y.; Lu, G.; Ma, W.; Wei, Z. Adv. Energy Mater. 2017, 7 (22), 1701548. doi: 10.1002/aenm.201701548

(46) Dai, S.; Zhao, F.; Zhang, Q.; Lau, T. K.; Li, T.; Liu, K.; Ling, Q.; Wang, C.; Lu, X.; You, W.; Zhan, X. J. Am. Chem. Soc. 2017, 139 (3), 1336. doi: $10.1021 /$ jacs.6b12755

(47) Yao, H.; Cui, Y.; Yu, R.; Gao, B.; Zhang, H.; Hou, J. Angew. Chem. Int. Ed. 2017, 56 (11), 3045. doi:10.1002/anie.201610944

(48) Ma, X.; Mi, Y.; Zhang, F.; An, Q.; Zhang, M.; Hu, Z.; Liu, X.; Zhang, J.; Tang, W. Adv. Energy Mater. 2018, 8 (1), 1702854. doi: 10.1002/aenm.201702854 